\title{
Las relaciones América Latina-U nión Europea: Antecedentes de la importancia e INSTITUCIONALIZACIÓN DEL DIÁLOGO POLÍTICO
}

\author{
Carlos D. Martin \\ instituto de Ciencia Politica, Pontificia Unimersidad Católica de Chile \\ Academia nacional de Estudios Políticos y Estratégicos (Anepe)
}

\begin{abstract}
Este artículo describe el progresivo aumento en la institucionalización de las relaciones políticas entre América Latina y la Unión Europea, apuntando a la creciente importancia de la región en el contexto mundial y la consolidación de la Unión Europea como un actor internacional unitario. Se sigue un orden cronológico, sobre la base de las distintas generaciones de acuerdos entre ambas partes. También se presenta una breve síntesis de los principales lineamientos de la visión común y planificación estratégica por parte de la UE como un actor unitario hacia AL. La relación muestra el camino hacia la asociación estratégica entre ambas regiones.
\end{abstract}

\section{INTRODUCCIÓN}

La relación existente entre Europa y América Latina (AL) tiene una larga tradición. Por más de 500 años AL ha estado en contacto con el viejo continente a través de sus vínculos comerciales y políticos. Los lazos culturales han evolucionado considerablemente desde la época de la Colonia. Europa ha estado atenta a los cambios en AL y, a su vez, esta última ha buscado constantemente establecer lazos políticos, económicos y culturales con Europa, que le permitan contrapesar la influencia de Estados Unidos, particularmente a partir del último siglo. Las relaciones entre Europa y América Latina han experimentado diversas etapas, siempre al interior de un marco de amistad y conducidas principalmente a nivel bilateral hasta la década de 1980 .

Por una parte, la importancia de AL en los asuntos internacionales ha aumentado constantemente, a pesar de algunos de los más críticos períodos económicos de la región. Por otra, la Unión Europea (EU) ha adquirido un papel más relevante como un actor internacional con una voz única que se levanta en la medida que su proceso de integración está siendo conformado y su política exterior común se hace más importante para sus Estados Miembros. Por ello, los intereses europeos en AL no son solo comerciales, sino también políticos y estratégicos.

El siguiente trabajo se centra en la descripción del proceso de institucionalización de la relación entre Europa y América Latina, principalmente lo referido a los aspectos políticos de ella. Los límites cronológicos, por lo tanto, son aquellos de la emergencia de la Comunidad Económica Europea, debido a que esta entregó los primeros mecanismos para institucionalizar la relación de los países europeos con los latinoamericanos. Adicionalmente a la introducción y conclusión, este documento se divide en otras cinco secciones. La primera comprende el periodo que se extiende desde 1958 hasta 1970 y muestra el comienzo de la relación. Esta es una época caracterizada 
por tratados comerciales no preferenciales y una persistencia de las relaciones bilaterales entre países. A continuación, la segunda, desde 1970 hasta 1979, se encuentra la década de los acuerdos comerciales de primera generación. En tercer lugar, sigue un período de Acuerdos Marco de Cooperación, 1980-1989, que corresponden a la segunda generación. Cuarto, los acuerdos de tercera generación son el eje de la sección para el período entre 1990 y 1994 en el cual fueron firmados. Finalmente, los acuerdos de cuarta generación comienzan en 1995 con el tratado firmado con Mercado Común del Sur, MERCOSUR, en 1995. Antes de las conclusiones generales de este trabajo, se sintetizan brevemente los principales lineamientos de la visión común y planificación estratégica por parte de la UE como un actor unitario hacia AL, a partir de algunos documentos comunitarios oficiales sobre la materia.

El desarrollo de la relación entre ambas regiones ha experimentado distintas etapas a través de su historia. Hasta la década de 1970, el énfasis de la relación era principalmente comercial. Sin embargo, a partir de los 1980s, Europa comenzó a adquirir mayor interés en los aspectos sociales y políticos de AL, demostrado especialmente con el respaldo al Proceso de Paz Centro Americano en la década de 1980. Este proceso fue en parte posible debido a la disminución en atención atribuida por Estados Unidos a Latinoamérica. La explicación de esta conducta radica en los impactos que la guerra de Vietnam, los rehenes en Irán y Watergate tuvieron en el interés y credibilidad de las actividades de Estados Unidos en los asuntos regionales. En este punto, y por una variedad de razones, los europeos asumieron un papel más activo en sus esfuerzos para alcanzar la estabilidad regional y consolidar la democracia en el continente. En términos generales, tradicionalmente la Unión Europea ha ofrecido apoyo al proceso de democratización, la defensa de derechos humanos y la integración de América Latina, ${ }^{1}$ aspectos que han sido formalmente institucionalizados a través del tiempo.

Las transformaciones internacionales de la década de 1990 facilitaron la profundización de un cambio cualitativo en la relación entre las dos regiones. Los procesos de transición hacia sociedades democráticas también contribuyó al interés de la UE para entregarle mayor institucionalización a su participación política en la región, una que estuviera más acorde con el potencial comercial y financiero de AL. Estas son manifestaciones de una preocupación global de la UE sobre la importancia estratégica de AL así como la consolidación y legitimización de una Política Exterior y de Seguridad Europea Común (PESC).

Antes de empezar con la descripción de las relaciones biregionales, es necesario establecer dos premisas centrales que orientan la estructura del presente trabajo en la materia. La primera es que en la medida que los eventos internacionales y las condiciones internas del propio proceso europeo de integración han tenido lugar, la Unión Europea ha requerido fortalecer su política exterior y de seguridad para participar en los asuntos internacionales como un "actor" unitario. ${ }^{2}$ Esto es un aspecto clave en torno al papel político y estratégico de la UE hacia AL.

1 Dromi, Roberto y Carlos Molina del Pozo, Acuerdo MERCOSUR-Unión Europea, Ediciones Ciudad Argentina, Buenos Aires, 1996, pp. 11 -12.

2 Esta premisa puede ser contraria a la comúnmente asumida dificultad para estudiar la Unión Europea como una sola unidad de análisis. Sin argumentar que ésta ya ha cambiado completamente, a partir de fines de la década de 1990 y la de 2000 es más fácil que en el pasado observar los esfuerzos de la UE para actuar como un actor unitario. 
La segunda premisa, intrínsecamente ligada a la anterior, es que las relaciones entre Europa y América Latina están alcanzando un punto de difícil retorno con la firma de los acuerdos de cuarta generación, debido a sus implicancias y consolidación. A pesar que las relaciones biregionales han estado dominadas principalmente por la retórica y los discursos diplomáticos, el nivel de institucionalización entre ambas regiones ha aumentado en momentos históricos claves. Esta institucionalización está experimentando un salto cualitativo a partir de mediados de la década de 1990, consolidando un diálogo político entre ambas regiones que es inherentemente vital para ambas, más allá de las consideraciones económicas. Para la UE, su relación política con Al es necesaria para una mayor consolidación de su Política Exterior y de Seguridad Común y su inserción en los asuntos mundiales como un "actor" internacional, más allá de su área tradicional de influencia. A su vez, para América Latina, las relaciones con Europa refuerzan su propio proceso de integración y democratización. Simultáneamente, los latinoamericanos intentan tener relaciones comerciales y de inversión con un mayor énfasis en el desarrollo que aquellas que se pueden obtener con Estados Unidos, las cuales aún están más marcadas por un tenor de seguridad.

\section{EL COMIENZO DE LA RELACIÓN, 1958-1969}

En el periodo pos-Segunda Guerra Mundial, las dos regiones comenzaron un proceso de institucionalización de sus relaciones. La situación latinoamericana estaba caracterizada por la progresiva importancia de su comercio y políticas en la arena internacional. Al mismo tiempo, Estados Unidos consolidaba su influencia en AL, llegando a ser efectivamente un poder hegemónico líder en asuntos internacionales.

Por muchos años la relación entre Europa y AL estuvo caracterizada por la existencia de relaciones bilaterales entre países individuales europeos y AL. En la década de 1950, con la progresiva consolidación de la Comunidad Europea, los vínculos biregionales comenzaron a adquirir mayor relevancia y ser institucionalizados.

En 1952 la Comunidad Europea del Carbón y Acero fue creada, probando ser una transformación fundamental en el contexto europeo que inició el proceso de integración. Hacia 1958 la emergente institucionalización europea fue fortalecida aún más con la Comunidad Económica Europea. El proceso de integración europeo recibió un impulso decisivo en 1967 con la creación de la Comunidad Europea, la cual unificó las instituciones existentes. En este momento, las relaciones internacionales de los Estados Miembros en común no eran una prioridad. Los miembros lentamente comenzaron a delegar algunas funciones en la Comunidad Europea en un delicado balance entre las atribuciones que los países delegaban y las que retenían para sí mismos. A pesar de esta característica de la Comunidad, que aún es una parte central de la Unión Europea que ha emergido a lo largo de los años, "[t]he creation of the European Community (EC) provided a mechanism through which the European states could deal collectively with other nations". ${ }^{3}$ La progresiva expansión de las relaciones exteriores todavía está en curso, particularmente con regiones extrahemisféricas como América Latina. 
En tanto, la integración latinoamericana estaba tomando forma hacia la década de 1960. Existen dos procesos que pueden ser destacados en este sentido. El primero es el Mercado Común Centro Americano (MCCA) formado en 1960. El segundo es la Asociación Latino Americana de Libre Comercio (ALALC), también de 1960.. Para fines de esta década un subgrupo de países andinos firmaron el Acuerdo de Cartagena, dando origen al Grupo Andino en 1969. Estos países eran Bolivia, Colombia, Chile, Ecuador y Perú. Venezuela se unió más tarde, pero Chile se retiró en 1974. Los países del caribe crearon la Asociación de Libre Comercio del Caribe (CARIFTA) por medio del Tratado de Antigua en 1968, la cual en 1970 se transformó en el Mercado Común del Caribe (CARICOM). CARICOM propuso posteriormente la creación de una Asociación de Estados del Caribe (ACS) para promover la integración y cooperación del caribe en general y reunir una gran variedad de países. La idea de la ACS estuvo circulando desde principios de la década de 1990, sin embargo se constituyó formalmente en 1994 en Cartagena de Indias y la primera reunión de jefes de Estado y Gobierno tuvo lugar en 1995.

El Tratado de Montevideo de 1980 transformó ALALC en ALADI, Asociación Latinoamericana de Integración, la cual junto con ser más flexible estaba orientada a la creación de un mercado común latinoamericano, consolidar el proceso alcanzado hasta el momento y fortalecer el carácter multilateral de la liberalización del comercio regional. Actualmente, uno de sus principales roles es el de avanzar en la convergencia de los diversos acuerdos en la región. De la ALADI emergieron dos subgrupos que han consolidado sus procesos de integración. El primero es el Mercado Común del Sur, MERCOSUR, de 1991, el cual, manteniendo vínculos legales con ALADI, tiene sus propias dinámicas. El segundo es el acuerdo que constituyó el Grupo de los Tres -Colombia, México y Venezuela- que se hizo efectivo en 1995, pero cuya idea había sido discutida desde 1989.

Dos instituciones deben ser mencionadas por su importante papel en el proceso de integración latinoamericano. La primera es la Comisión Económica para América Latina y el Caribe (CEPAL), 1948, con sede en Santiago-Chile. Y segundo, el Sistema Económico para América Latina (SELA) formado en 1975 con el Acuerdo de Panamá y localizado en Caracas, Venezuela, pero abierto a todos los países de la región. Ambas instituciones promueven la integración en la región y el diálogo con otras áreas.

\section{Relaciones entre la Unión Europea y América Latina}

En términos de la relación bilateral entre la UE y AL, Mower diferencia entre distintas fases. Durante la primera, correspondiente al período entre 1958 y 1963, la CE produjo un "Memo de Intensión" para los estados latinoamericanos. En este punto, la Comunidad manifestó que su objetivo formal era alcanzar una "estrecha relación y cooperación con América Latina" y buscó disipar los temores en AL respecto a la posibilidad que las preferencias de la CE por el comercio interno fueran a dañar las propuestas comerciales latinoamericanas. A su vez, debido a sus preocupaciones respecto del comercio, los latinoamericanos crearon ALALC para reforzar el comercio y tam- 
bién emitieron declaraciones indicando que la CE constituía una "casi guerra en las proyecciones de exportaciones Latino Americanas". ${ }^{5}$

Una segunda fase en las relaciones biregionales se extiende desde 1963 hasta 1967. En este período, la CE creó vínculos más estrechos con sus ex colonias en África (Acuerdos de Yaounde), brindándoles un trato preferencial. Los latinoamericanos se volvieron más aprehensivos respecto a su acceso a los mercados de la CE. En este contexto, se realizaron dos series de conversaciones entre la CE y AL. La CE les solicitó una lista de las exportaciones latinoamericanas que, desde su perspectiva, estaban siendo afectadas. Asimismo, la CE buscó una declaración por parte de AL sobre sus aspiraciones comerciales de largo plazo.

A través de sus representantes diplomáticos en Bruselas, los latinoamericanos respondieron produciendo "El Memorando Latino Americano de 1966". Este memo contiene objetivos específicos y una declaración respecto de la necesidad de una política económica interregional comprensiva y propone una comisión permanente conjunta compuesta por representantes de la CE y jefes de las misiones latinoamericanas en la Comunidad. Sin embargo, de acuerdo a Mower y otros autores como Durán, no hubo respuesta por parte del Consejo de la CE, el cual tampoco reaccionó a un informe posterior de la Comisión de la CE sobre la materia. ${ }^{6}$

En consecuencia, durante la tercera fase, 1967-1970, las relaciones bilaterales estaban en un punto extremadamente bajo, siendo los principales dos hechos la inactividad del Consejo y la virtual suspensión de todos los contactos oficiales entre la CE y AL. Un estudio del Directore General IA de la Unión Europea realizado para una pasantía, indica el año 1970 como el punto de inflexión en el cual una relación caracterizada solo por "algunos intercambios informales de opinión" comenzó a cambiar con la publicación de la Carta de Buenos Aires de la Comisión Especial de Coordinación Latinoamericana (CECLA), la cual fue creada para las relaciones con la Comisión Europea. $^{7}$

De acuerdo al autor de este informe de la UE, aquel documento, que realiza un llamado a un diálogo político y económico entre las dos regiones, fue exitoso en el sentido que generó en la Comisión un impulso a las relaciones con AL. ${ }^{8}$ Drekonja-Kornat también entrega un buen recuento de este tiempo, como un período en el cual las esperanzas de muchos latinoamericanos en los primeros años de la Comunidad Económica Europea rápidamente desaparecieron hasta $1970 .{ }^{9}$

7 Directore General IA, Acuerdos Bilaterales y Relaciones entre la UE y Latinoamérica, IA/337/95-ES, Bruselas, julio 1995, p. 7. Más precisamente, el CECLA fue un grupo ad hoc creado en 1963 para coordinar las actitudes de los países latinoamericanos en los temas que tratarían en la primera reunión de la UNCTAD. Durán, Esperanza, European Interest in Latin America, The Royal Institute of International Affairs, 1985, p.12.

8 Directore General, ibid., 1995, p. 7. El autor cita una Comunicación al Consejo "Las Relaciones con los países Latinoamericanos" de 1969 como una prueba de la reacción de la UE a la Carta de Buenos Aires. Sin embargo, las fechas no se correlacionan adecuadamente; de hecho, la Comunicación es un año anterior a la Carta. De todas maneras, el argumento que las relaciones bilaterales cambiaron sustancialmente puede ser sostenido, como lo hace Mower también, entre otros aspectos por el flujo de información y versiones no oficiales del documento que serían conocidas.

9 Drekonja-Kornat, Gerhard, "Western Europe-Latin America: A general frame of reference", en Angarita, Ciro and Peter Coffey, Europe and the Andean Countries: A comparison of economic policies and institutions, Pinter Publishers, London \& NY, 1988. A pesar de no ser el centro de su argumento, tampoco el de su libro, en este capítulo el autor ofrece una buena elaboración de algunos de los aspectos de las relaciones biregionales alrededor de 1970. Específicamente pp. 7-13. 
En referencia a la institucionalización de una relación política entre ambas regiones, se aprecia un avance casi nulo en este sentido para el período en cuestión. Si bien existen intentos para encontrar o diseñar mecanismos adecuados de relacionamiento, el eje son los esfuerzos para revertir los perjuicios, reales o percibidos, en términos comerciales, que la integración europea tendría sobre la región latinoamericana.

\section{ACUERDOS COMERCIALES, 1970-1979}

La Declaración de Buenos Aires de julio de 1970, que básicamente hace un llamado a retomar los contactos oficiales entre las dos regiones y al establecimiento de una comisión conjunta permanente, produjo una reacción positiva en el Consejo de la CE hacia junio de 1971, cuando comenzó una serie de conversaciones regulares semi-anuales a nivel de embajadas. ${ }^{10}$ Este es el Diálogo de Bruselas, que reúne al Grupo de Embajadores en Bruselas (GRULA) y los representantes de la CE. ${ }^{11}$ Durante el período entre 1970 y 1976, las relaciones bilaterales se incrementaron sostenidamente y se concluyeron acuerdos no preferenciales entre la CE y Argentina en 1971, Uruguay en 1973, Brasil en 1973 y México en 1975. Los tratados de este período eran acuerdos comerciales no preferenciales, por lo cual recibieron el nombre de Acuerdos de Primera Generación. Whitehead indica que estos fueron los primeros acuerdos de cooperación que la Comisión Europea comenzó a negociar con países en vías de desarrollo, aparte de aquellos cubiertos por la Convención de Lomé de 1975. ${ }^{12}$

En referencia a las relaciones comerciales existen algunos acuerdos para demostrar estas relaciones más cercanas. Durán, sin embargo, afirma que esta opción de acuerdos bilaterales fue adoptada por la CEE debido a una falta de progreso en el Diálogo de Bruselas..$^{13}$ A pesar de este punto, los tratados tienen una considerable importancia para $\mathrm{AL}$, que incluso se extiende más allá de relaciones bilaterales. Los cuatro tipos de acuerdos mencionados por Mower son, primero, Acuerdos Comerciales Bilaterales entre la UE y Argentina, Brasil y Uruguay. Segundo, el Pacto de Cooperación Económica con México. Tercero, acuerdos particulares tales como aquellos sobre textiles con Argentina, Brasil, Colombia, Guatemala, México, Perú y Uruguay. Finalmente, también algunos Arreglos Especiales de cuotas para ciertos productos artesanales. En este caso los arreglos involucran a Uruguay, Brasil, Ecuador, Paraguay, Honduras, Perú y El Salvador.

En general sin embargo, la mayoría de la atención de la CE estaba en los países cubiertos por la Convención de Lomé o aquellos en el sistema de asistencia preferencia (ACP, por sus siglas en inglés). nos. De hecho, él es más específico, e indica que el grupo era liderado por Gabriel Valdés, ministro de Relaciones Exteriores de Frei Montalva. Drekonja-Kornat, ibid., P. 8.

11 Los autores concuerdan en que estas reuniones no contribuyeron significativamente de inmediato a la creación de relaciones más cercanas entre ambas regiones, tal es el caso de Durán, op cit., 1985 and Drekonja-Kornat, ibid., 1970. Sin embargo, se puede sostener que en el largo plazo ellas contribuyeron a los posteriores acuerdos de comercio y cooperación. Este también es un punto algo más fuerte que el hecho en la época por Mower, op. cit., 1982.

12 Whitehead, Laurence, "The European Union and the Americas", en Victor Bulmer-Thomas and James Dunkerley, The United States and Latin America: The new agenda, ILAS, 1999, p. 55. La Convención de Lomé contiene, sucesivamente desde 1975, el marco de las ayudas de comercio y cooperación de la Comunidad con unos 70 de los países menos desarrollados de África, el Caribe y el Pacífico. 
Al mismo tiempo, debe ser reconocido que este es el período en el cual el sistema generalizado de preferencias comenzó a consolidarse, el cual hasta hoy en día es central en la mayoría de las negociaciones entre ambas regiones. En 1977 el Comité de Representantes Permanentes (COREPER) y el Grupo-Latinoamericano (GRULA) comenzaron a tener reuniones regulares. Sin embargo, ellas fueron interrumpidas en 1982 como consecuencia del conflicto de las Islas Falkland/Malvinas y no se reanudaron sino hasta 1989.14 Durán también se refiere a este esfuerzo de restituir el Diálogo de Bruselas, pero en general parece menos optimista de sus resultados. Aparte de la guerra de 1982, la autora también agrega la admisión de Cuba al GRULA en 1979, especialmente complicada porque fue el año de la invasión de Afganistán por parte de la entonces Unión Soviética. ${ }^{15}$

En paralelo a los acuerdos comerciales, las reuniones Inter-Parlamentarias comenzaron en 1974. Este hecho representa una de las manifestaciones más importantes de la institucionalización de las relaciones biregionales en el ámbito político. A partir de esa fecha, las reuniones Inter-Parlamentarias entre el Parlamento Europeo y el Latino Americano han tenido lugar cada dos años, representando una de las principales avenidas de las relaciones interregionales.

Una publicación de CELARE sobre el papel del Parlamento Europeo en el fortalecimiento de las relaciones bilaterales distingue tres periodos en la participación del Parlamento Europeo en AL. La primera, denominada apertura, comienza en la década de 1960 hasta mediados de la de 1970. La segunda está relacionada con el desarrollo de los vínculos políticos y económicos entre las dos regiones, así como con la lucha por la paz y los derechos humanos en los '80s. El siguiente período se extiende hasta nuestros días y se relaciona con la cooperación en asuntos sobre el futuro y el apoyo a la transición y consolidación democrática. ${ }^{16}$ La conclusión de este libro es que el Parlamento ha jugado un papel gatillador en las relaciones bilaterales, demostrando la importancia que los parlamentarios le atribuyen a las relaciones políticas y su institucionalización debido a las preocupaciones con la situación en AL. ${ }^{17}$ Un aspecto importante de considerar en relación con el Parlamento Europeo es el hecho que, desde 1979, sus miembros han sido elegidos directamente; esto puede ser una fuente de mayor involucramiento del Parlamento en materias políticas en AL.

En términos de las relaciones políticas entre Europa y $\mathrm{AL}$, el fortalecimiento progresivo de los vínculos bilaterales puede ser adicionalmente considerado a través del estatus de observador permanente que la Comunidad Europea tiene en varias organizaciones regionales, tales como SELA, ALADI, OEA y la Junta del Acuerdo de Cartagena (UUNAC). ${ }^{18}$ A lo largo del tiempo, se diseñaron distintos arreglos institucionales o técnicas de manejo de las relaciones bilaterales para facilitar la revisión de los patrones existentes de cooperación, la generación de sugerencias de resolución de controversias y la proposición y discusión de oportunidades para nuevas formas de cooperación, tales como el Diálogo de Brus elas y otras instancias o visitas oficiales. En esta línea,

Directore General IA, op. cit., 1995, p. 8. El autor, sin embargo, no entrega información respecto al trabajo/temas de estas reuniones.

15 Durán, op., cit., 1985, p. 13.

16 CELARE, El Parlamento Europeo y América Latina: El rol del Parlamento Europeo en el fortalecimiento de las relaciones biregionales, 1996. p. 11. Otra buena fuente de información al respecto es IRELA, El Diálogo Interparlamentario Comunidad Europea-América Latina, 1974-1992: Un examen de las relaciones interregionales, Documento de Trabajo, $\mathrm{N}^{\circ} 36$, 1993.

17 CELARE, ibid., 1996, p. 83.

18 Dromi, op., cit., 1996, p. 14. 
se puede destacar el hecho que la UE decidió tener mayor presencia en la región abriendo más oficinas en ella ${ }^{19}$.

La ayuda de la UE dirigida hacia AL estaba orientada hacia la integración y la transferencia de recursos. Un primer ejemplo era el amplio flujo total de recursos financieros externos hacia AL. ${ }^{20}$ Un segundo aspecto, resaltado por Mower, es el creciente monto de ayuda de la UE, el cual se duplicó desde el 10\% que representaba en 1976 a 1979. Desde el punto de vista de la UE, este aumento en la ayuda dirigida a AL tiene el objetivo de alcanzar un mejor balance geográfico en la ayuda que entrega en el mundo. En tercer lugar, la UE también dirigió fondos hacia Al a través del Banco de Desarrollo Inter-Americano (BID), al cual miembros de la UE se unieron durante este período. ${ }^{21}$ También se esperaba que a través de su participación en el BID, el trato comercial con la región se viera favorecido, en áreas tales como la contribución al flujo de inversiones privadas en los negocios de AL. Adicionalmente, algunos recursos fueron enviados a la región por la vía del Banco de Inversión Europeo. Finalmente, y a pesar de la percepción de su papel controvertido, las Corporaciones Multinacionales también han sido un medio importante de transferencia de recursos a la región.

La UE alentó estos arreglos porque prefirió relacionarse con las naciones de una manera colectiva y también debido a que, de acuerdo a su propia experiencia, esta era la mejor manera de mejorar las posibilidades de intercambio entre grupos de países, lo que a su vez beneficiaría a la UE. El proceso también fue una consecuencia de la decisión en 1974 de otorgarle asistencia financiera y técnica a países fuera de la Convención de Lomé, al interior de la prioridad de programas que promovían la cooperación e integración regionales. La UE también autorizó al Banco de Inversión Europeo a ampliar sus programas de ayuda para incluir a AL. Adicionalmente, la CE también apoyó la integración latinoamericana a través de su colaboración con ALALC, MCCA y el Grupo Andino a través de las instituciones para la integración regional. Finalmente, se hicieron modificaciones a los principios de las "reglas de origen" que tenían la intención de promover el comercio biregional.

En suma, el énfasis en el establecimiento de relaciones estuvo más en el lado de AL y el diálogo establecido solo eventualmente contribuyó a la futura institucionalización de las relaciones biregionales. De hecho, durante este período las relaciones entre estas dos áreas permanecieron estando muy dominadas por las relaciones bilaterales entre Estados miembros de la UE y países individuales de AL. Para Mower, en la década de 1970 Europa y AL tuvieron relaciones más cercanas comparadas con las de épocas anteriores, ${ }^{22}$ pero se puede agregar que esta cercanía

19 Sin embargo, la oficina de la Comisión en Santiago fue cerrada y trasladada a Caracas debido a las preocupaciones respecto a los derechos humanos en Chile.

20 Mower, op. cit., 1982, p. 53-54. De hecho, el coeficiente mencionado por Mower es el de 16\% para el período 1951 1956, 28\% para 1978, y un considerable salto a 40\% para 1975.

21 En relación a la decisión de incorporarse al BID, Mower menciona un punto interesante respecto a que existieron consideraciones respecto a unirse o no a una institución donde participaba un país con el récord de derechos humanos como el de Chile. Sin embargo, la idea de entregar asistencia tangible a una institución de asistencia fue privilegiada.

22 Mower, op. cit., 1982, p. 38. Para explorar este argumento la información puede ser dividida en tres grandes áreas funcionales de relación entre la UE y AL: Comercio, Asistencia, y Técnicas de relaciones bilaterales. Para Mower, gran parte de la década de 1970 fue un período de cercanía y lejanía, en el cual los principales avances en las relaciones bilaterales fueron las experiencias adquiridas en una amplia gama de temas y el reconocimiento del potencial de la relación entre la UE y AL. 
facilitó la posterior decisión política de participar en la resolución del conflicto en América Central y, a su vez, fomentar la institucionalización de las relaciones bi-regionales.

\section{ACUERDO MARCO DE COOPERACIÓN, 1980-1989}

En referencia a las relaciones entre Europa y AL, Esperanza Durán afirmó en 1985 que era "hailed for their promise and growing importance. However, hard facts and achievements have not yet kept pace with the political pronouncements and expressions of goodwill made by political leaders in both regions". ${ }^{23}$ En un argumento muy similar al de Drekonja-Kornat, Durán describe los mismos elementos en la relación bilateral, enfatizando que hasta mediados de la década de 1980, fecha en la cual ella estaba escribiendo, no hubo un mejoramiento significativo de las relaciones a pesar del hecho que la Comunidad introdujera un nuevo mecanismo colectivo para las relaciones exteriores.

A pesar de las afirmaciones generales, los acuerdos de este período representan un impulso a las relaciones biregionales. Estos son los acuerdos de segunda generación. El primero es firmado con Brasil en 1980, luego con los países andinos en 1983 y América Central en 1985. Estos dos últimos son una demostración del interés particular de la Comisión Europea por potenciar las subregiones por sobre países individualmente. ${ }^{24}$

En forma adicional a estos acuerdos, un aspecto más importante de destacar aún es la creciente institucionalización de las relaciones políticas. Además de los acuerdos de cooperación comercial, en 1984 se inició el Proceso de San J osé. Este diálogo representa una institución que todavía es importante para las relaciones entre Europa y AL. Para Whitehead, el diálogo "was a major new departure in that it involved high level political mediation, it was long-term and contractual in character, and it committed the community as a collectivity to the promotion of peace and reconstruction in Central America". ${ }^{25}$ El autor también destaca que esta iniciativa no discriminatoria, en constraste a la posición de Estados Unidos, fue una "unusual and highly political initiate [that] was undertaken by a joint agreement between all members of the European Community", ${ }^{26}$

Las relaciones bilaterales recibieron un impulso adicional en esta década con el ingreso de España y Portugal a la Comunidad Europea en 1985. Roy y Galinsoga construyen el argumento que España trató de recobrar su presencia en AL después de su propio retorno a la democracia y su nueva política exterior ligada a la incorporación a la Unión Europea. ${ }^{27}$ El argumento de los autores, que no considera plenamente el historial de las relaciones, es que Europa había participado ocasionalmente en los asuntos de AL, esto especialmente válido en términos de seguridad, pero también en general, sosteniendo que la influencia de la región en términos políticos había sido muy limitada.

24 La idea de una preocupación regional comparada a las individuales es destacada en: Directore General, op. cit., 1995, p. 10.

25 Whitehead, op. cit., 1999, p. 55. El autor está usando material de J oaquín Roy, "The identity of the new Europe and the San José Process" en The Reconstruction of Central America: The role of the European Union. North-South Center, University of Miami, 1992.

26 Whitehead, ibid., 1999, p. 55.

27 Roy, J oaquín y Albert Galinsoga J ordà (eds) The Ibero-American Space: Dimensions and perceptions of the special relationship between Spain and Latin America, Iberian Studies Institute, University of Miami; J ean Monnet Chair for European Integration, University of Lleida, 1997. Estas ideas están en el capítulo introductorio escrito por los editores, pp. 1-6. 
Más aún, que su influencia económica era dispareja y que sus acciones culturales eran tímidas y a medias, alcanzando solo algunas elites intelectuales. Específicamente en relación a la influencia de España en la región, se considera que la restricción que España tiene en este período es la limitada experiencia y recursos de ayuda para el desarrollo. Adicionalmente, se destaca "the lack of public, and specially private, Spanish investment in Latin America" ${ }^{28}$ son los principales factores que explican el bajo nivel de participación española en la región en esos momentos. De acuerdo a los autores, la falta de interés Europeo en AL se complementa con estas condiciones en España para "an uneven repercussion and an asymmetric position for Spanish initiates in Latin America". ${ }^{29}$

En un estudio más reciente y completo, Schumacher se refiere al vínculo entre España y AL. Uno de sus argumentos centrales es la formación de una comunidad Ibero-Americana que ha sido benéfica para ambas partes. La reaparición de una relación especial entre ambas ha significado beneficios considerables para España en términos de la internacionalización de su economía y el fortalecimiento de su posición política en el mundo, particularmente al interior de la UE y con Estados Unidos. ${ }^{30}$ La detallada descripción que Schumacher hace de la evolución de la Hispanidad, entrega evidencia sobre algunos elementos claves de la relación entre Europa y AL, que se mencionan para este período, tales como el Diálogo de San J osé, que fue el mayor test de España como un puente entre las dos regiones. ${ }^{31}$ En suma, "[t]he European Union is the single most important instrument of Spanish foreign policy in Latin America. It is also the resource in which Latins are most interested." ${ }^{\prime 32}$ Las altas inversiones realizadas en la década de 1990 por las compañías españolas, tanto en Chile como en otros países de la región, son evidencias de este argumento.

La participación de la UE en el conflicto de América Central fue un elemento clave para la resolución de este. Para la UE representa un gran logro político. A lo largo de la década de 1980 "decade (in what is probably Europe's greatest foreign policy success to date), the EC articulated perceptions of the crisis that were characterized by the view that the conflict" era más bien un asunto Norte-Sur que Este-Oeste. Este énfasis en las causas internas del conflicto armado estaban en claro contraste con el enfoque adoptado por Estados Unidos. ${ }^{33}$ Otra manifestación del interés político de Europa en AL puede ser observada en la activa participación de la UE en la Conferencia Internacional sobre los Refugiados en América Central (CIREFCA). Desde 1989 hasta

Roy, ibid., 1997, p. 3. El Comité de Inversiones Extranjeras indica para el período 1974-2000 a España como segundo principal inversionista individual.

29 Roy, ibid., 1997, p. 3

30 Schumacher, Edward, Spain and Latin America: The Resurgence of a Special Relationship, en Kaufman Purcell, Susan and Françoise Simone, Europe and Latin America in the World Economy, 1995, p. 113. Por ejemplo, este tema se hizo bastante evidente en las inversiones españolas en Chile, y otros países de la región, en sectores como los de energía y telecomunicaciones en años recientes.

31 Schumacher, ibid., 1995, p. 118. El autor también menciona que España fue el primer país en respaldar el Proceso de Paz de Contadora, además de otras actividades conducidas por la diplomacia española durante esa década.

32 Schumacher, ibid., 1995, p. 119. Mayor información sobre el papel de España puede ser encontrada en: Rosenzweig, Gabriel, España y las Relaciones entre las Comunidades Europeas y América Latina, IRELA, Documento de Trabajo, $N^{\circ} 8$, 1987.

33 Crawley, Andrew, "Toward a biregional agenda for the twenty-first century", en Journal of InterAmerican Studies and World Affairs, Vol. 42, N. 2, Summer 2000. P. 12. Este es un número especial titulado The European Union and Latin America: Changing relations, editora invitada Alexandra Barahona de Brito. 
1994 la UE trabajó para ayudar a las personas refugiadas, repatriadas y desplazadas en la región. ${ }^{34}$ Estos hechos muestran la creciente importancia para la UE de actuar como un solo actor en asuntos internacionales y además, en el caso de latinoamericano, exitosamente.

\section{ACUERDOS MARCO DE COOPERACIÓN COMERCIAL Y ECONÓMICA, 1990-1994}

Basado en la experiencia del Diálogo de San José, en 1991, se institucionalizó un nuevo Diálogo en 1991, esta vez con el Grupo de Río. Ahora los países de América del Sur y México tuvieron la oportunidad de disponer de vínculos directos con Europa y viceversa. Los diálogos políticos institucionalizados con el Grupo de Río experimentaron un cambio cualitativo en la IV Reunión Ministerial de Río en abril de 1994 cuando ésta concluyó con una declaración conjunta sobre los temas económicos, comerciales, industriales, científicos y tecnológicos. ${ }^{35}$

En parte por su papel político y, por otra, fomentado también por algunas de las transformaciones internacionales que estaban ocurriendo en ese momento, el Consejo de la UE aprobó en 1990 las "Orientaciones para la Cooperación con los Países en Vías de Desarrollo de América Latina y Asia" (PVDALA). ${ }^{36}$ Este documento representa una institucionalización formal de la previa cooperación más bien informal existente con la región de AL. Al mismo tiempo, este estableció una diferenciación no solo entre países y diferentes regiones, tales como Asia y $\mathrm{AL}$, sino que también entre países más y menos desarrollados en una misma región, lo cual permite la aplicación de programas de cooperación mejor adaptados a las necesidades particulares de los países de acuerdo a su nivel específico de desarrollo. ${ }^{37}$

Durante este período correspondiente a la primera mitad de la década de 1990, las relaciones biregionales en el plano político se profundizaron notablemente. Las dos regiones firmaron algunos acuerdos de cooperación, así como algunos más particulares entre países individualmente. Estos son acuerdos denominados de tercera generación, debido a que incluyen las cláusulas democrática y evolutiva. La cláusula democrática es un manifiesto apoyo de la UE a los procesos democráticos y de derechos humanos en AL. Básicamente significa que la UE tiene el derecho a revocar su asistencia de cooperación en caso de reversión del proceso democrático regional. La cláusula evolutiva implica que formas adicionales de cooperación pueden ser convenidas entre las Partes sin necesidad de volver a negociar un acuerdo completo.

Los acuerdos firmados en estos años son: Argentina el 2 abril 1990; Chile el 20 diciembre 1990; México en 1991; y Uruguay en 1991. En este periodo la Unión Europea comienza las transformaciones del Tratado de Maastricht, 1992, y a pesar de ellas los acuerdos con AL continuaron, como Paraguay en 1992. También en 1992, MERCOSUR y la CEE firmaron un Acuerdo de Cooperación Inter-Institucional. Los otros acuerdos son Brasil en 1992, América Central en 1993 y el Grupo Andino en 1993.

Durante esta década también se destacan las Conferencias Iberoamericanas. Esta instancia representa una sustancial institucionalización permanente del diálogo político y de cooperación entre ambas 
regiones. A partir de 1991, en la Cumbre de Guadalajara, se comienza con las reuniones anuales de los Jefes de Estado y Gobierno, de habla portuguesa y castellana, como una forma de incrementar la reflexión e intercambio de ideas conjunto sobre diversos temas en el marco de este diálogo bi-regional.

La expansión de estos tipos de acuerdos es importante para la región porque ésta enfrenta dos procesos muy diferentes. Por una parte se encuentra la consolidación del proceso democrático iniciado en años anteriores en algunos países. ${ }^{38}$ Por la otra, existe una necesidad de profundizar las reformas económicas, considerando las dimensiones sociales del desarrollo así como el desarrollo sustentable. Este es el contexto en el cual emergió "an upsurge in mechanisms for biregional cooperation and political dialogue". ${ }^{39}$ El contexto político que se venía desarrollando en AL desde la década de 1980 alentó la participación de la UE en este de acuerdo a la tradición y valores apoyados por la Comunidad, siendo innovadora la implementación de la cláusula democrática en los arreglos de comercio y cooperación a nivel mundial. ${ }^{40}$

\section{ACUERDOS DE CUARTA GENERACIÓN, 1995-2000}

La iniciativa de firmar estos acuerdos de asociación de cuarta generación comenzó con MERCOSUR en diciembre de 1995, "Acuerdo Marco Interregional de Cooperación UE/MERCOSUR". El siguiente fue firmado con Chile en 1996, "Acuerdo Marco de Cooperación UE/Chile". México alcanzó otro el 8 de diciembre de 1997, "Acuerdo de Asociación Económica, Concertación Política y Cooperación UE/México". De hecho, México, fue el primer país latinoamericano en firmar un Acuerdo de Libre Comercio con la UE el 23 de marzo del 2000, el cual entró en vigor el 1 de julio del mismo año.

Para el SELA, la presencia de diferentes esquemas de acuerdos (desde preferenciales hasta de cuarta generación) firmados por la UE con distintas subregiones en el continente pueden ser una fuente de complicaciones y dificultades para instrumentalizar las relaciones bilaterales. ${ }^{41}$

Los acuerdos de cuarta generación se caracterizan por los siguientes elementos: asociación económica, coordinación y cooperación política, acuerdos de reciprocidad e interés mutuo, institucionalización del diálogo político, liberalización del comercio de bienes y servicios (de acuerdo a las normas de la OMC) de manera bilateral y preferencial así como progresiva y recíproca, respeto por principios democráticos y de derechos humanos, creación de un Consejo Conjunto, cooperación en diversos sectores como el industrial, inversiones financieras, Pequeñas y Medianas Empresas (PYMES), cláusula evolutiva, acuerdos sobre contrataciones públicas, políticas de competencia y derechos de propiedad, y diálogo político conducido en diferentes niveles (presidencial,

De hecho la idea de la cláusula democrática emergió de los negociadores chilenos (Mariano Fernández) del acuerdo bilateral entre la Comunidad Europea y Chile debido a las condiciones específicas existentes en nuestro País. A partir de las negociaciones con Chile, esta cláusula fue incorporada en otros acuerdos negociados en la región. El acuerdo de la UE con Argentina, en el cual aparece dicha cláusula, fue firmado unos pocos meses antes del alcanzado con Chile debido a que el proceso para la firma fue más rápido en ese país que en Chile. Agradezco a Rene Muga por las distintas entrevistas sobre estas materias en ProChile-Londres, por ejemplo, esta del 29 de marzo de 2001.

39 Crawley, op. cit., 2000, p. 13.

40 Para mayor información sobre este aspecto ver por ejemplo, Toro, Sergio, "Acuerdos entre la Unión Europea y Terceros Países sobre la Cláusula Democrática o de Derechos Humanos", en Revista Diplomática, N ${ }^{0} 77$, octubre-diciembre 1998.

41 SELA, Reflexiones entorno a la Cumbre Unión Europea-América Latina y el Caribe, 1999, (SP/DRE/Di N²4-28), p. 1. 
ministerial, altos oficiales), entre otros aspectos. ${ }^{42}$ En lugar de la asistencia en la manera tradicional, los acuerdos de cuarta generación implican mejores posibilidades de acceder a los programas intercomunitarios de investigación y desarrollo, formación/ educación, tecnologías avanzadas y telecomunicaciones. ${ }^{43}$

En paralelo a estos eventos biregionales, la UE realizó una importante evolución. El primer aspecto que debe ser mencionado es el Tratado de Amsterdam en 1997. Otro hito fundamental fue el Tratado de Niz en 2000, el más largo hasta el momento. Estos procesos de institucionalización de la UE, algunos de ellos particularmente importantes en torno a la política exterior de la UE y sobre algunas de las decisiones adoptadas por ellos al interior del GATT, luego OMC, también condicionan la relación entre la UE y AL. Adicionalmente, la discusión sobre ampliación o profundización al interior de la UE tiene mucho impacto en las relaciones exteriores de la Comunidad.

Un documento de la Comisión Europea, "The European Union and the World", describe el papel que la UE juega en los asuntos internacionales, comenzando con la premisa que la UE "is working to ensure a stable and peaceful Europe with a voice that is heard in the world". ${ }^{44}$ En referencia a AL el documento establece que la UE reconoce tres subgrupos regionales (América Central, la Comunidad Andina y MERCOSUR) y dos países individualmente (Chile y México). La evaluación de la década de 1990 es que las relaciones biregionales "have consistently developed as bilateral trade has expanded and the Union has promoted regional integration in the area". ${ }^{45}$ Además de remarcar las relaciones con el Grupo Andino y México, la Primera Reunión de los J efes de Estado y de Gobierno de la UE y Al y el Caribe, en Río de J aneiro en 1999, es considerada "a quantum leap in the Union's relations with this part of the world". ${ }^{46}$ La siguiente demostración del nivel de compromiso y probabilidad en la relación bilateral puede ser encontrada en la Cumbre de Madrid entre las dos regiones en mayo de 2002. ${ }^{47}$ Las Cumbres representan un punto de partida, sin embargo, en la búsqueda de mecanismos de largo plazo en las relaciones biregionales. Ellas han demostrado ser una buena oportunidad para desarrollar políticas y compromisos que puedan beneficiar a todos los actores involucrados. En particular desde el punto de vista latinoamericano, las relaciones económicas/comerciales con Europa son de trascendental importancia. Las tendencia comerciales ente ambas regiones también están en el fondo de la búsqueda de un diálogo, en un sentido general y no solo político, más fluido entre las partes, el cual incluya los mecanismos adecuados para referirse a las importantes disputas en esta materia ente ambas regiones. ${ }^{48}$

SELA, ibid., 1999, p. 1.

Hacia 1995 IRELA detallaba estas ventajas, IRELA, La nueva Europa y su impacto en América Latina, Dossier N ${ }^{0}$ 54, marzo 1995, sin embargo existe un nivel más adelantado de diálogo político avanzado por el concepto de "alianza estratégica" entre las dos regiones. Este elemento no era mencionado con frecuencia a comienzos de este período. Comisión Europea, Information brochure for the general public, Europe on the Move, dec. 2000, p. 1.

Comisión Europea, ibid., dec. 2000, p. 19.

Comisión Europea, ibid., dec. 2000, p. 19.

CELARE se refiere a este tema, entre otros. Por ejemplo, ver el texto: CELARE, Primera Cumbre América Latina y el Caribe - Unión Europea. Una reflexión Política Estratégica, CELARE, Santiago, 1998. Sobre el ámbito político en particular se puede ver el artículo de Gabriel Gaspar, pp. 93-123.

Algunos planteamientos sobre esta materia se pueden ver en: Leiva, Patricio (ed.), Una Asociación Estratégica para e Siglo XXI, CELARE, Santiago, 1999. En particular e incluyendo los aspectos comerciales de la relación son expresados en el mismo libro por Mariano Fernández, CELARE, Primera Cumbre América Latina y el Caribe-Unión Europea. Una reflexión Política Estratégica, CELARE, Santiago, 1998, pp. 167-194 
El argumento principal de este trabajo es que, al mismo tiempo, se ha avanzado en la consolidación de un diálogo político que trasciende los temas solo biregionales. Esto es la consolidación de una asociación estratégica entre ambas regiones en la cual, además de los temas tradicionales como las relaciones comerciales y el refuerzo del proceso democrático y de derechos humanos en Latinoamérica, se está buscando primar el funcionamiento conjunto en la sociedad internacional.

En general, los eventos durante toda esta década emergen como una demostración de un gran esfuerzo de institucionalización de las relaciones políticas entre las dos regiones. Existen tres niveles en los cuales se pueden organizar las relaciones biregionales. El primero comprende las cuatro generaciones de acuerdos mencionadas anteriormente, incluyendo el acuerdo recientemente alcanzado entre la UE y Chile. Un segundo nivel es representado por instrumentos previamente institucionalizados de diálogo entre ambas áreas. Estos son, por una parte, el Diálogo de San José y, por otra, las Reuniones Ministeriales con el Grupo de Río y las anuales de J efes de Estado y Gobierno Iberoamericanos. Así mismo, en tercer lugar, las Reuniones Inter-Parlamentarias han continuado durante este periodo y han cumplido un papel importante en la realización de las Cumbres entre la UE y AL y el Caribe, así como el fortalecimiento de las relaciones políticas entre las dos regiones. ${ }^{49}$ Finalmente, en los últimos años ha emergido una nueva forma de institucionalización, representada por el Diálogo Político avanzado subregional o bilateralmente, el cual tiene una alta expresión en las Cumbres de J efes de Estado y Gobierno de las dos regiones. La Cumbre de Río en 1999 y la de Madrid en 2002 han sido una plataforma en la cual los temas biregionales son discutidos y se adoptan resoluciones que tienen un fuerte impacto en el desarrollo de esta asociación estratégica biregional.

\section{VISIÓN Y PLANIFICACIÓN ESTRATÉGICA DE LA UNIÓN EUROPEA, 1995-2000}

La creciente importancia de AL en los asuntos mundiales alienta la noción en la UE de fortalecer sus relaciones con la región. La voluntad de la UE para establecer nuevos niveles de institucionalidad en las relaciones biregionales con AL ha sido particularmente evidente desde la segunda mitad de la década de 1990. La realización de las Cumbres de Jefes de Estado y de Gobierno de ambas regiones le han otorgado una fluidez adicional en años recientes, facilitando instancias a través de las cuales se ha reforzado la noción de una asociación estratégica en distintos niveles. ${ }^{50}$ El desarrollo de esta voluntad se plasma en distintos documentos al respecto.

En términos de programación estratégica se destacan dos documentos. Primero, la Comunicación de la Comisión titulada The European Union and Latin America. The Present Situation and Prospects for Closer Partnership. 1996-2000, de 1995. Segundo Latin American Regional Strategic Document, 2002-2006 programming, de 2002. En la comparación de ambos documentos se constata la evolución desde una asociación más centrada en los temas latinoamericanos, tales como el proceso de paz en la región, democratización y derechos humanos, y el hecho de poder

49 El punto aquí está basado en declaraciones generales, visitas y reuniones así como en acciones específicas tales como aquellas llevadas a cabo en 1998 respecto al tema de la Ley de Amnistía de Pinochet, su categoría de miembro permanente del Congreso y su detención.

50 Por ejemplo, ver documento Comunicación Comisión (COM 670/2000), Seguimiento de la primera cumbre celebrada entre América Latina, el Caribe y la Unión Europea. 2000. 
aportar en ellos hacia otras aspiraciones de carácter internacional como la concertación en foros mundiales.

El primero de los trabajos, un documento orientador sobre las prioridades de la UE respecto a sus relaciones bilaterales con $\mathrm{AL}$, divide temáticamente los vínculos en tres áreas: institucionalización del diálogo, reuniones Inter-Parlamentarias, y acuerdos de Cooperación. El principal argumento respecto a estas áreas es que "[t]he steadily improving quality of political relations between the European Union and Latin America over recent years is borne out by the institution of a formal regional and sub-regional political dialogue". ${ }^{51}$

Posteriormente el segundo documento se centra, en términos de prioridades, en las acciones que se pueden realizar para que junto con los aspectos latinoamericanos se pueda apoyar la "nueva asociación estratégica" entre ambas regiones. ${ }^{52}$ En este sentido, los programas de cooperación delineados por el documento se centran, primero, en las medidas tendientes a reforzar la sociedad civil. Segundo, iniciativas sociales que contribuyan a disminuir la desigualdad en la región. Luego se contemplan acciones para prevenir las catástrofes naturales y reconstrucción. Finalmente, se menciona el observatorio de las relaciones biregionales, como una medida complementaria a esta asociación estratégica en curso.

La institucionalización del diálogo político entre ambas regiones es tratada desde el primer documento en 1995, el cual destaca la creación de una "maquinaria" política estable construida a partir del Diálogo de San José y el Diálogo con el Grupo de Río desde 1990.53 Las reuniones InterParlamentarias son resaltadas como un instrumento que ha permitido a las dos regiones desarrollar este diálogo político sobre la base de temas comunes y conservando los énfasis sobre las necesidades latinoamericanas. Finalmente, en referencia a los acuerdos de cooperación, se establece que desde 1990 se ha realizado un esfuerzo para firmar acuerdos de tercera generación con toda la región (exepto Cuba).

Para el documento publicado en 2002 se reconoce el diálogo político entre ambas regiones resaltado anteriormente, pero no pone el énfasis sobre ellos. Esto representa un reconocimiento a la importancia de las Cumbres, Río y Madrid, y a cómo se debe trabajar para orientar las acciones de cooperación o de la relación biregional en general hacia el fortalecimiento de la asociación estratégica entre ambas. En cuanto a la firma de acuerdos, se puede resaltar que actualmente el eje radica en los de cuarta generación, como el que ya cuenta la UE con México y siendo el alcanzado con Chile el más reciente de éstos.

Otro tema importante es que además de las formas tradiciones de cooperación, se han sumado otras en áreas nuevas en los últimos años. En general, en este sentido, las orientaciones genera-

51 Commission Communication (CAB III/164/95-EN), ibid., 1995. Específicamente en la sección "1. Substantial progress in the political dialogue". A pesar de no estar explicitado en el informe, este parece ser el Informe consensuado producido por J an-William Bertens, no sin algo de dificultades.

52 European Commission, Latin American Regional Strategic Document, 2002-2006 programming, 2002.

53 En referencia al Diálogo con el Grupo de Río, el documento destaca un aspecto ya mencionado en este trabajo, que la "joint declaration adopted at the fourth ministerial meeting, held in São Paulo in April last year (1994), underlines the climate of partnership that now characterizes relations between the two regional groupings". Commission Communication (CAB III/164/95-EN), ibid., 1995. 
les para las relaciones biregionales establecidas por las instituciones de la Comunidad para el período 1991-1995, representan una consolidación y mejoramiento de lo que se podría denominar cooperación tradicional. Estas áreas incluyen la ayuda humanitaria, programas de desarrollo rural, integración regional, y desarrollo del sector de recursos marinos. ${ }^{54}$ Para el período proyectado entre 2002 y 2006, los ámbitos de actividad radican en el apoyo a las redes de la sociedad civil, iniciativas sociales para disminuir las desigualdades, enfrentar las catástrofes naturales, y el observatorio de las relaciones regionales.

A partir de principios de la década de 1990, las nuevas áreas de cooperación para la Comisión tienen "a greater emphasis on long-term and sustainable development and the use of instruments which promote economic modernisation" del que tenían estrategias previas de cooperación. ${ }^{55}$ Esto es importante de destacar ya que es consistente con el argumento construido en la planificación estratégica para este período respecto a la creciente institucionalización de las relaciones biregionales, especialmente sobre los instrumentos económicos y políticos.

El énfasis de la Comisión radica en tres áreas. La primera es la de democratización y derechos humanos, lo cual se refiere básicamente a los esfuerzos de la UE de ser un actor activo en los esfuerzos para contribuir a la transición democrática a un Estado de derecho y la participación de la sociedad civil en el desarrollo de una cultura de derechos humanos en AL. En segundo lugar se encuentran los proyectos de asistencia financiera y técnica, principalmente programas y proyectos urbanos, los esquemas para promover los derechos y libertades fundamentales de las mujeres y su plena integración en el proceso de desarrollo, y los proyectos orientados a mejorar las condiciones de vida de los pueblos indígenas y el respeto por su identidad cultural y educación o formación. También se incluyen proyectos y programas para proteger el medio ambiente (manejo de bosques tropicales, contaminación urbana, entre otros), de acuerdo con los compromisos de la UE para asignar al menos $10 \%$ de su asistencia en AL a proyectos medioambientales. ${ }^{56}$ Finalmente, se manifiesta el apoyo económico para las actividades de mutuo interés en ciencia y tecnología, las cuales han aumentado considerablemente desde $1980 .{ }^{57}$

J unto al mejoramiento de las formas tradicionales de cooperación y la inclusión de nuevos temas, también se constata el deseo de implementar nuevos métodos para la cooperación biregional, tales como la diversificación de socios, estrategias por país y sectores, programas horizontales, y evaluaciones sistémicas. ${ }^{58}$

Uno de los temas centrales de la estrategia sugerida por la Comisión para fortalecer las relaciones con AL se refiere a los aspectos políticos. El argumento para el nuevo impulso a las relaciones biregionales de la UE se basa en el recientemente adquirido papel de AL en el sistema internacional. El diálogo biregional debe incluir tanto el ámbito Ibero-Americano como los foros internacionales. ${ }^{59}$ 
Un mayor desarrollo de la posición adoptada por las instituciones de la UE puede ser apreciado en el Parlamento europeo. El Parlamento acogió favorablemente el documento de la Comisión, respondiendo con una resolución adoptada el 16 de junio de 1997. A pesar de su apoyo a las propuestas del documento, este solicitó un programa de acción que incluyera "propuestas concretas". 60 En el Boletín de la Resolución, el Parlamento manifiesta que "economic cooperation agreements with countries and regional groupings be accompanied by financial protocols with substantially greater amounts allocated for cooperation, that they focus on economically and socially sustainable development and that they bring a reciprocal and negotiated opening-up of markets". ${ }^{61}$ Al mismo tiempo se hace un llamado para eliminar parte de la deuda externa de algunos países e integrar mejor a las sociedades civiles.

Sin embargo, lo más significativo en esta resolución puede ser encontrado en referencia a la declaración sobre los aspectos de política exterior de las relaciones biregionales. El Parlamento "wished to see Latin America figure more prominently in the common foreign and security policy and security and defence cooperation, and for a new form of transatlantic dialogue, modelled on that with the United States and Canada, to be set up for Latin American countries".62 Este es un elemento importante porque puede indicar algunas de las orientaciones adoptadas por uno de los actores más relevantes de la institucionalización de las relaciones biregionales, así como el modelo que éstas pueden seguir.

Adicionalmente, El Consejo también se adhiere a las conclusiones generales del Comunicado de la Comisión. El Consejo destaca su voluntad de fortalecer los vínculos políticos, apoyar la democracia, conducir procesos de liberalización comercial, apoyar los procesos de integración regional, y mejorando las orientaciones de la cooperación de la UE. Estas son las bases de "deepening the institutionalized dialogue with the Latin American interlocutors". ${ }^{63}$ Para alcanzar estos objetivos el Consejo define tres ejes de prioridades institucionales. Primero, apoyo institucional a los procesos democráticos en la región, incluyendo la protección de los derechos humanos. Segundo, lucha contra la pobreza y la exclusión social. Tercero, reformas económicas. ${ }^{64}$

Las prioridades que la UE establece en su relación con AL han experimentado una evolución paulatina desde fines de la década pasada y los primeros años de la actual. En términos de cooperación se busca profundizar el apoyo a la sociedad civil y las desigualdades presentes en la región. También resaltan temas específicos sobre catástrofes naturales y recuperación. Sin embargo, el principal respaldo lo recibe la asociación estratégica y la idea que las acciones de la UE contribuyan en esta dirección, reforzándose el concepto del diálogo político como un eje prioritario de las relaciones biregionales y el posicionamiento de la UE como un actor internacional.

60 Parliament Resolution [0] C 33, 3.2.1997], Bulletin EU 1 / 2 -1997, Latin America (1/8).

61 Parliament Resolution [O] C 33, 3.2.1997], Bulletin EU 1 / 2 -1997, Latin America (1/8).

62 Parliament Resolution [O] C 33, 3.2.1997], ibid., 1997.

63 Consejo, Proyecto de conclusiones del Consejo relativas a las orientaciones generales para la cooperación entre la Comunidad y América Latina 1996-2000, 12153/95, 1995, p. 1.

64 Consejo, 12153/95, ibid., 1995, p. 3-4. 


\section{CONCLUSIONES}

El nivel de institucionalización de las relaciones políticas entre la Unión Europea y América Latina ha aumentado progresivamente a lo largo de las décadas. El manejo de las relaciones comerciales, aún presente, tuvo una prioridad casi exclusiva alrededor de la década de 1960, cuando las relaciones biregionales tenían en un bajo nivel.

El primer acercamiento significativo se constata durante la década de 1970, cuando, por ejemplo, se firman los acuerdos de primera generación y comienzan las reuniones Inter-Parlamentarias. Con posterioridad, las relaciones han estado caracterizadas por sucesivas generaciones de acuerdos, de comercio y cooperación, estrechamente relacionados con la evolución del contexto internacional. A través de ellos se empiezan a plasmar diversos aspectos políticos.

En la década de 1980, el ingreso de España y Portugal a la Comunidad facilita un impulso adicional a las relaciones biregionales. También se constata el inicio de diálogos políticos específicos y un muy importante papel de la UE en la resolución del conflicto Centroamericano. La noción de una UE como un "actor" internacional es patente hacia fines de este período.

Para los acuerdos de tercera generación, a principios de la década de 1990, dos cambios cualitativos fueron introducidos: las cláusulas democráticas y las evolutivas, constatándose una profundización de las relaciones políticas entre ambas regiones, como se puede apreciar en las Conferencias lberoamericanas. En la segunda mitad de la década pasada existe un claro esfuerzo por institucionalizar el diálogo político entre ambas regiones. Esto es nuevo y único en su relación.

Este proceso no solo ha sido promovido por las experiencias bilaterales pasadas, tales como el Diálogo de San José y las Reuniones Inter-Parlamentarias, sino también por una necesidad en ambas regiones de pensar la relación mutua tanto por requerimientos internos en cada una de ellas como en términos de estratégias internacionales. En el caso de la UE, la consolidación de su papel como un actor internacional y el desarrollo de su Política Exterior y de Seguridad Común son partes esenciales del análisis. Para AL Europa representa una gran ayuda en términos de asistencia para el desarrollo. Más importante es, sin embargo, el hecho que esta relación es un pilar para sus propios procesos de democratización e integración. Ambos temas refuerzan el potencial de $\mathrm{AL}$ en la arena mundial.

Estratégicamente, ambas regiones están pensando en su posicionamiento internacional. Ninguno de los dos puede permitirse ignorar al otro si quiere reforzar su propia postura internacional. La institucionalización del diálogo político y su alianza estratégica es crítica en el actual contexto internacional en el cual existe una constante búsqueda de nuevas normas y reglas para conducir los asuntos mundiales.

\section{BIBLIOGRAFÍA}

CELARE, El Parlamento Europeo y América Latina: El rol del Parlamento Europeo en el fortalecimiento de las relaciones biregionales, CELARE, Santiago, 1996.

CELARE, Primera Cumbre América Latina y el Caribe -Unión Europea. Una reflexión Política Estratégica, CELARE, Santiago, 1998. 
COMMISSION COMMUNICATION, The European Union And Latin America. The Present Situation And Prospects For Closer Partnership. 1996-2000, (CAB III/ 164/95-EN), Brussels, 1995.

COMISIÓN COMUNICACIÓN (COM 670/2000), Seguimiento de la primera cumbre celebrada entre América Latina, el Caribe y la Unión Europea. 2000.

CRAWLEY, ANDREW, "Toward a biregional agenda for the twenty-first century", in Journal of Internamerican Studies and World Affairs, University of Miami, Miami, Vol. 42, №2, Summer 2000. Special issue The European Union and Latin America: Changing relations, guest editor Alexandra Barahona de Brito.

DROMI, ROBERTO AND CARLOS MOLINA DEL POZO, Acuerdo MERCOSUR-Unión Europea, Ediciones Ciudad Argentina, Buenos Aires, 1996.

DIRECTORE GENERAL IA, Acuerdos Bilaterales y Relaciones entre la UE y Latinoamerica, IA/337/95-ES, Brussels, July 1995.

DREKONJ A-KORNAT, GERHARD, "Western Europe-Latin America: A general frame of reference", in Angarita, Ciro and Peter Coffey, Europe and the Andean Countries: A comparison of economic policies and institutions, Pinter Publishers, London \& NY, 1988.

DURÁN, ESPERANZA, European Interest in Latin America, The Royal Institute of International Affairs, London, 1985.

EUROPEAN COMMISSION, Information brochure for the general public, Europe on the Move, Dec. 2000.

EUROPEAN COMMISSION, Latin American Regional Strategic Document, 2002-2006 programming, 2002.

IRELA, El Diálogo Interparlamentario Comunidad Europea-América Latina, 1974-1992: Un examen de las relaciones interregionales, Documento de Trabajo, № 36, Madrid, 1993.

IRELA, La nueva Europa y su impacto en América Latina, Dossier № 54, Madrid, marzo 1995.

LATIN AMERICAN ECONOMIC SYSTEM, 1999 Guide to Latin American and Caribbean Integration, SELA Electronic Book, Caracas, 1999.

LEIVA, PATRICIO (ed.), Una Asociación Estratégica para el Siglo XXI, CELARE, Santiago, 1999.

MOWER, A. GLENN, The European Community and Latin America: A case study in global expansion, Greenwood Press, West port, 1982.

Parliament Resolution [OJ C 33, 3.2.1997], Bulletin EU-1997, Latin America (1/8), Brussels, 1997.

ROY, J OAQUÍN, "The identity of the new Europe and the San J osé Process" in The Reconstruction of Central America: The role of the European Union. North-South Center, University of Miami, 1992.

ROY, JOAQUÍN AND ALBERT GALINSOGA JORDÀ (eds) The Ibero-American Space: Dimensions and perceptions of the special relationship between Spain and Latin America, Iberian Studies Institute, University of Miami; Jean Monnet Chair for European Integration, University of Lleida, 1997.

ROSENZWEIG, GABRIEL, España y las Relaciones entre las Comunidades Europeans y América Latina, IRELA, Documento de Trabajo, № 8, Madrid, 1987

RENE MUGA, ProChile-Londres, Londres, 29 marzo 2001.

SELA, Reflexiones entorno a la Cumbre Unión Europea-América Latina y el Caribe, (SP/DRE/Di No 24-28), Caracas, 1999.

SELA, 1999 Guide to Latin American and Caribbean Integration, SELA Electronic Book, 1999.

SCHUMACHER, EDWARD, Spain and Latin America: The Resurgence of a Special Relationship, en Kaufman Purcell, Susan and Françoise Simone, Europe and Latin America in the World Economy, Boulder, Colo. London: Lynne Rienner, 1995.

TORO, SERGIO, “Acuerdos entre la Unión Europea y Terceros Países sobre la Cláusula Democrática o de Derechos Humanos", en Revista Diplomática, № 77, Santiago-Chile, octubre-diciembre 1998.

WHITEHEAD, LAURENCE, "The European Union and the Americas", in Victor Bulmer-Thomas and James Dunkerley, The United States and Latin America: The new agenda, LAS, London, 1999. 